\title{
Tactile perception in blind Braille readers: A psychophysical study of acuity and hyperacuity using gratings and dot patterns
}

\author{
ARTHUR C. GRANT, MAHESH C. THIAGARAJAH, and K. SATHIAN \\ Emory University School of Medicine, Atlanta, Georgia
}

\begin{abstract}
It is not clear whether the blind are generally superior to the sighted on measures of tactile sensitivity or whether they excel only on certain tests owing to the specifics of their tactile experience. We compared the discrimination performance of blind Braille readers and age-matched sighted subjects on three tactile tasks using precisely specified stimuli. Initially, the blind significantly outperformed the sighted at a hyperacuity task using Braille-like dot patterns, although, with practice, both groups performed equally well. On two other tasks, hyperacute discrimination of gratings that differed in ridge width and spatial-acuity-dependent discrimination of grating orientation, the performance of the blind did not differ significantly from that of sighted subjects. These results probably reflect the specificity of perceptual learning due to Braille-reading experience.
\end{abstract}

Deprived of sight, the blind negotiate their external world with the nonvisual senses, primarily hearing and touch. The notion that their increased dependence on the auditory and somatosensory systems may be associated with supernormal perceptual abilities in these modalities has been investigated for at least a century (Griesbach, 1899; Hollins, 1989). In the somatosensory system, recent work has confirmed earlier findings (reviewed by Hollins, 1989) that von Frey and two-point discrimination thresholds are no different in the blind and the sighted (Pascual-Leone \& Torres, 1993). Similarly, discrimination of the texture of sandpaper is performed equally well by congenitally blind, adventitiously blind, and normally sighted subjects (Heller, 1989b).

Braille reading is a skill that distinguishes the blind. The Braille code consists of a 6 -cell, $3 \times 2$ rectangular matrix, with dots being present in some or all of the cells for a given character. Although it was initially proposed that Braille pattern recognition is based on shapes outlined by the dots encoding each character (Loomis, 1981), extensive work with both sighted and blind subjects led instead to the conclusion that the critical cues are textural, related to variations in dot spacing or density (Millar, 1994).

The findings in this report were presented at the 27th Annual Meeting of the Society for Neuroscience, New Orleans, LA (Grant, Thiagarajah, Chevalier, \& Sathian, 1997). This work was supported in part by Grant R29NS34111 from the NINDS to K.S. The authors thank Andro Zangaladze for help with testing and for preparing Figure I, Sarah Chevalier for help with testing and for comments on the manuscript. Gargi Patel for help with testing, and the staff of the Center for the Visually Impaired, especially Debbie Page and Deborah Hudson, and Jim Cashun of the Georgia Radio Reader for help with subject recruitment. Most of all, the authors are grateful to the subjects for their willing participation. Correspondence should be addressed to K. Sathian, Depariment of Neurology, Emory University School of Medicine, WMRB6000, P.O. Drawer V, Atlanta, GA 30322 (e-mail: ksathia(@)emory.edu).
Blind Braille readers were better than sighted controls at tactile recognition of dot patterns (with similar dot diameter and spacing to Braille) presented in $3 \times 3$ and $4 \times 4$ matrices but not $5 \times 5$ or $6 \times 6$ matrices (Foulke \& Warm, 1967). It was thought that the Braille-reading experience of the blind subjects accounted for these findings, due to carryover specifically to the smaller patterns, which could be entirely covered by the fingertip, whereas the larger patterns required sequential exploration.

Another tactile reading method used by some blind people employs the Optacon, consisting of an array of probes in which a pattern of stimulation on the fingerpad is generated from scanning of a hand-held camera across print. This device was used to present stimuli in a letter identification task that was familiar to previous Optacon users and an unfamiliar gap detection task (Craig, 1988). Two groups of blind Braille readers were studied, one group being users of this device and the other without prior experience with it; their performance on both tasks was similar. Among sighted subjects, a group with some Optacon experience did as well as the blind subjects, but an Optacon-naive group performed poorly by comparison. These results were taken as evidence that tactile experience, regardless of its specifics, leads to a general heightening of tactile sensitivity. The generalizability found in this study, which appears to conflict with the findings with large dot matrices (Foulke \& Warm, 1967), could well reflect the similarity between the tasks used and also between patterns of stimulation on the skin generated by Braille and Optacon reading.

On more complex tasks involving haptic perception of shape, reported findings include poorer performance of blind subjects relative to sighted subjects (Bailes \& Lambert, 1986; Lederman, Klatzky, Chataway, \& Summers, 1990), similar performance in both groups (Morrongiello, Humphrey, Timney, Choi, \& Rocca, 1994), superiority 
of the blind over the sighted (Heller, 1989a), and an advantage for those with late-onset blindness over those with early-onset blindness or normal vision (Heller, 1989a). Kennedy (1997) observed that drawings and haptic interpretations of perspective by the blind and the sighted reflect broadly similar perceptual organization in these two groups, although each group appears to have advantages on particular aspects of perspective (Heller, Calcaterra, Tyler, \& Burson, 1996; Heller, Kennedy, \& Joyner, 1995). It has been argued that any advantage of the blind on haptic tasks reflects, not heightened sensitivity, but the results of practice in attending to cues that normally are ignored by the sighted (Hollins, 1989) or the use of more efficient sensorimotor strategies (D'Angiulli, Kennedy, \& Heller, 1998; Davidson, 1972; Shimizu, Saida, \& Shimura, 1993).

Use-dependent plasticity at the level of the cerebral cortex has been documented in blind individuals. Somatosensory evoked potentials and transcranial magnetic stimulation (TMS) in blind Braille readers demonstrate an expanded cortical sensory representation of the Braillereading finger (Pascual-Leone \& Torres, 1993), motor output maps of which expand and contract dynamically as a function of the amount of Braille reading in the hours prior to derivation of the maps by TMS (Pascual-Leone, Wasserman, Sadato, \& Hallett, 1995). Although these cortical changes might be expected to confer an overall increase in tactile sensitivity, the evidence reviewed above is equivocal in this regard.

Gratings and dot patterns, which are stimuli offering precise control over spatial dimensions, have been well characterized in a number of psychophysical and neurophysiologic studies of the tactile system and have contributed importantly to our understanding of tactile perception (Sathian, 1989). We therefore investigated, psychophysically, how blind Braille readers and age-matched sighted controls performed on three distinct tactile tasks, one employing Braille-like dot patterns and the other two utilizing gratings. Discrimination performance was measured in all three tasks; form recognition was not called for in any of them. We were interested in distinguishing between three possible outcomes: (1) Performance of the blind and the sighted would be comparable on all these tasks, indicating that there is no general superiority of tactile acuity in the blind. (2) The blind would outperform the sighted on a subset of the tasks, indicating specific advantages due to prior tactile experience. (3) The blind would excel on all three tasks, consistent with a general superiority of tactile acuity. Outcomes 2 and 3 could reflect use-dependent cortical plasticity.

\section{GENERAL METHOD}

\section{Subjects}

Blind subjects were recruited through a local center for the visually impaired or an audio bulletin board. Sighted subjects with normal or corrected-to-normal visual acuity were recruited from among students and staff at Emory University or its affiliated hospitals. All subjects provided written informed consent and were paid at an hourly rate for their participation. Procedures were approved by the
Human Investigations Committee of Emory University. Handedness of the sighted subjects was determined using the 14 items of the Edinburgh handedness questionnaire with the highest validity (Raczkowski, Kalat, \& Nebes, 1974), and handedness of the blind subjects was determined based on seven of these items that retained relevance for blind individuals.

Thirty-nine healthy, neurologically normal right-handed subjects served as sighted controls (Tables 1-3). Their ages ranged from 19 to 75 years $($ mean $=39.0)$. Each sighted subject did not necessarily run in all three tasks. The mean age of the sighted group was almost identical to the mean age of the blind subjects. Tables 1-3 also show that there was little difference in the mean ages of sighted subjects completing each task.

A total of 24 blind subjects, all Braille readers and ranging in age from 18 to 70 years $($ mean $=40.5$ ), were recruited. Most but not all participated in all three tasks (Table 4). No blind subject had vision that was better than light perception. Twenty were right-handed, and 4 were left-handed. Fourteen subjects were congenitally blind, and 1 subject had become totally blind before the age of 5 ; these 15 subjects were classified as early-blind subjects. Nine subjects had become totally blind after the age of 10 ; these subjects were classified as late-blind subjects. Five of them had significant visual impairment (but with some preservation of form vision) prior to complete loss of vision. The cause of blindness was variable, ' but none of the subjects had any other neurologic impairment. There was very little difference in the mean ages of early- and late-blind subgroups (Table 4). The duration of blindness at the time of testing ranged from 23 to 67 years for early-blind subjects and from 12 to 37 years for late-blind subjects. The rationale for the distinction between early and late blind is the well-known critical period in vision: Although its duration in humans is not clearly defined, it is generally accepted to last 5-10 years postnatally (Adams \& Victor, 1993). During this critical period, the visual system is capable of much greater plasticity than later, as revealed by the classic neurophysiologic studies of Hubel and Wiesel (1977) in cats and monkeys.

Exclusion criteria for all subjects included a history of neurologic disorders, brain injury, peripheral neuropathy, trauma to the fingers or their innervation, and the presence of fingerpad calluses. ${ }^{2}$ For sighted subjects, a history of learning disability (in particular, dyslexia) was also exclusionary, since dyslexics have been shown to be perceptually impaired on two of the three tactile tasks used in this study (Grant, Zangaladze, Thiagarajah, \& Sathian, 1999).

\section{Determination of Discrimination Thresholds}

Tactile testing was performed with stimuli screened from view of the sighted subjects. The psychophysical method used in all experiments was the method of constant stimuli. Discrimination thresholds were computed by linear interpolation between the values spanning threshold, as determined from psychometric functions. Further details are given in the Method sections of the individual experiments.

\section{Statistical Testing}

Analyses of variance (ANOVAs) were performed using SAS. The general linear models (GLM) procedure was used with Type III sums of squares. When a significant effect was found, post hoc Scheffé testing was used to assess the significance of pairwise differences in means. In addition, paired (two-tailed) $t$ tests were used in certain instances. The $\alpha$ value was .05 in all cases.

\section{EXPERIMENT 1 Hyperacute Discrimination of Dot Patterns}

In Experiment 1, embossed dots similar to those used in Braille were used. It was predicted that, owing to the similarity of the stimuli to Braille dots, the Braille-reading experience of the blind would facilitate their performance 
on this task, resulting in supernormal thresholds. However, in the present experiment, subjects had to make a perceptual judgment based on the presence of an offset of the central dot in a row of three (Figure 1A); they were not asked to make a symbolic interpretation (as occurs with Braille) or to identify particular patterns (as required in other studies using Braille-like dot matrices as stimuli). Moreover, they were required to touch the dots without any lateral scanning motion, as is typically used in Braille reading. These factors could potentially offset the advantage of familiarity with the stimuli.

The task used in this experiment is a hyperacuity taskthat is, one in which the threshold is finer than spatial resolving acuity (Loomis, 1979; Westheimer, 1977), which in turn corresponds to the receptor spacing in both vision and touch (Phillips, Johnson, \& Browne, 1983). The limit of tactile spatial resolution in the human fingerpad under conditions of static touch (i.e., without lateral scanning) is about $1 \mathrm{~mm}$ (Johnson \& Phillips, 1981; van Boven \& Johnson, 1994a). Neurophysiologic studies in monkeys indicate that the receptors defining this limit are the slowly adapting Type I (SAI) or Merkel afferents (Phillips \& Johnson, 1981) and that spatial shifts in the profile of activity within the SAI afferent pool most likely underlie static tactile hyperacuity (Wheat, Goodwin, \& Browning, 1995). In our hyperacuity task, repeated transient tactile contacts were permitted, so that a contribution from the rapidly adapting (RA) or Meissner afferents cannot be completely excluded (Sathian \& Zangaladze, 1998). In the human fingerpad, the innervation density of both SAI and RA afferents is approximately 1 per square millimeter (Johansson \& Vallbo, 1979). This implies a spacing of about $1 \mathrm{~mm}$, which matches the acuity of spatial resolution.

\section{Method}

The stimuli and task were adapted from a previous study of tactile hyperacuity (Loomis, 1979). The standard stimulus was a row of three embossed dots of about $0.3 \mathrm{~mm}$ in diameter, with a centerto-center spacing of approximately $2 \mathrm{~mm}$ (Figure 1A, left). Com- parison stimuli consisted of similar patterns in which the central dot was offset laterally (Figure 1 $\mathrm{A}$, right) by amounts varying from 0.1 to $1 \mathrm{~mm}$. Complete details of the spatial parameters and method of preparation are given in an earlier report (Sathian \& Zangaladze, 1998). These dot patterns were formed on plastic, steel-backed plates, which were mounted during the testing sessions into magnetic holding wells. The subject was asked to bring the index fingerpad down vertically onto the patterns, which were oriented along the long axis of the finger with the offset (if present) always to the right. As in Loomis's (1979) study from which the present task was adapted, scanning movements were prohibited. It is well known that lateral scanning improves tactile performance in textural tasks (Krueger, 1970; Meenes \& Zigler, 1923; Morley, Goodwin, \& Darian-Smith, 1983), probably due to increases in the firing rate of SAI afferents along with recruitment of purely dynamic mechanoreceptor types (Johnson \& Lamb, 1981; Phillips et al., 1983). Therefore, we avoided scanning movements to minimize the chance of encountering performance ceiling effects that could have masked any superiority in the blind.

In each trial, the subject compared a standard stimulus and a comparison stimulus presented side by side. The task was to state whether the stimulus with the offset was on the right or the left. The subjects were free to go back and forth between the patterns as many times as they wanted, without time limits. Trials were presented in blocks of 10 , with a different comparison stimulus in each block. The discrimination threshold was taken as the offset corresponding to $75 \%$ correct. Blocks spanning the threshold were repeated, so that threshold determinations were usually based on 30-40 trials for the most relevant offsets. Both hands of the blind subjects were tested in each session, with the dominant hand tested first. Only the dominant hand of the sighted controls was tested.

Previous work in our laboratory has demonstrated that there is considerable perceptual learning with this task, the threshold typically dropping by about 50\% over a few sessions (Sathian \& Zangaladze, 1998). It was of interest whether any performance difference between the blind and sighted subjects when they were task-naive would persist after practice. Hence, we tested most subjects in four sessions that were at least a day apart. An initial threshold was computed from the first session and a final threshold by pooling performance values across the last two sessions, between which there was no significant threshold difference. ${ }^{3}$

\section{Results}

Thresholds for the sighted subjects are shown in Table 1 and for the blind subjects in Table 4 (DP columns). Fig-
A

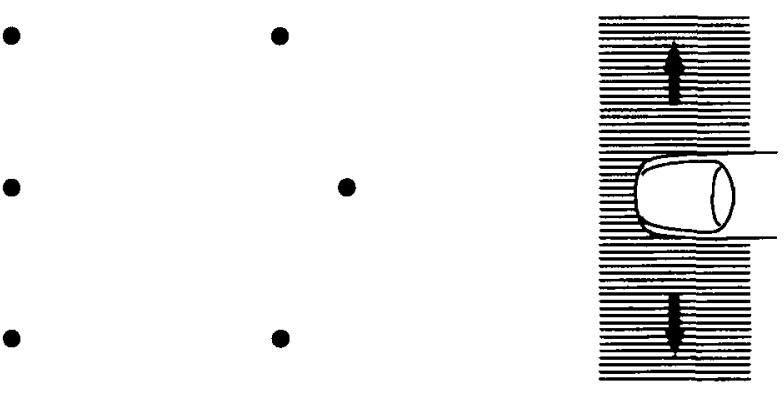

C
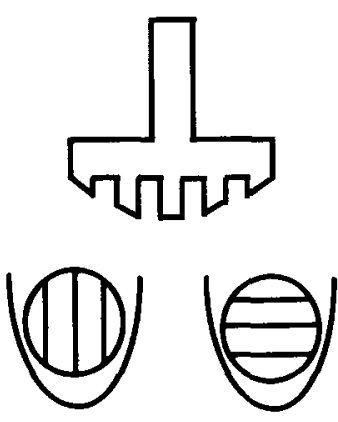

Figure 1. Lllustration of tactile stimuli used. (A) Discrimination of a dot pattern in which the central dot is offset laterally (right) from a standard pattern without such offset (left). (B) Discrimination of grating ridge width (RW) using active scanning. (C) Discrimination of the orientation of gratings applied to the passive fingerpad (above, cross-sectional profile of grating; below, diagrammatic representation of grating oriented along [left] or across [right] the fingerpad). 
Table 1

Data for the Sighted Subjects on Dot Pattern Discrimination

\begin{tabular}{ccccc} 
& & & \multicolumn{2}{c}{ Threshold $(\mathrm{mm})$} \\
\cline { 5 - 6 } Subject & Age (years) & Gender & Initial & Final \\
\hline S12 & 53 & F & 0.68 & 0.60 \\
S13 & 39 & M & 0.70 & 0.53 \\
S14 & 32 & M & 0.70 & 0.30 \\
S15 & 23 & F & 0.42 & 0.23 \\
S17 & 31 & F & 0.35 & 0.16 \\
S20 & 22 & F & 0.50 & 0.30 \\
S27 & 53 & F & 0.35 & 0.18 \\
S30 & 53 & F & 0.58 & 0.25 \\
S34 & 48 & F & 1.00 & \\
S36 & 21 & M & 0.43 & 0.17 \\
S37 & 29 & M & 0.87 & 0.57 \\
S38 & 27 & F & 0.35 & 0.14 \\
S39 & 34 & M & 0.58 & 0.35 \\
M & 35.8 & & 0.58 & 0.32 \\
SEM & 3.4 & & 0.06 & 0.05 \\
\hline
\end{tabular}

ure 2 shows the grouped performance data on the dominant hand. The blind subjects had considerably lower initial thresholds than the sighted subjects. Compared with the average initial threshold for sighted subjects, the average initial threshold for early-blind subjects was approximately half and that for late-blind subjects was about a third lower (Figure 2). Experience-related learning on this task was substantial for the sighted subjects; a smaller magnitude of improvement was seen in the early-blind subjects, whereas the late-blind subjects showed no trend to improve with practice. Figure 2 shows that, as a result, final thresholds were comparable among subject groups.

Since the sighted subjects were tested only on the dominant hand, statistical analysis was done in stages. A oneway ANOVA showed a significant effect of group [control, early blind, and late blind; $F(2,30)=4.42, p=.02]$ on the initial threshold on the dominant hand. Post hoc Scheffé testing revealed that the mean initial (dominant hand) thresholds were significantly different between sighted and early-blind groups. The thresholds for the late blind, being intermediate between those of the other two groups (Figure 2), did not differ significantly from either of them on the Scheffé test. A repeated measures ANOVA on the initial thresholds of the blind subjects showed no significant effect of subgroup [between subjects; early vs. late blind; $F(1,16)=1.02, p=.33$ ] or hand [within subjects; dominant vs. nondominant; $F(1,16)=0.13, p=$ .73] and no significant interaction $[F(1,16)=0.07, p=$ $.8]$. Since, in some cases, a subject's preferred Braillereading finger was not on the dominant hand, we compared initial performance on the index finger of the preferred versus nonpreferred hand for Braille-reading, using a paired $t$ test across the entire group of blind subjects, but found no significant difference $(p=.35)$. Final thresholds on the dominant hand did not differ significantly by group, according to a one-way ANOVA $[F(2,24)=0.87$, $p=.43$ ], nor was there a significant effect of subgroup $[F(1,9)=0.02, p=.88]$, hand $[F(1,9)=1.06, p=.33]$, or interaction $[F(1,9)=0.77, p=.4]$ on the subsequent repeated-measures ANOVA for the blind subjects.

\section{Discussion}

As expected, the blind subjects had superior performance relative to the normally sighted subjects, but this difference was present only for the initial threshold. The early-blind subjects clearly outperformed the sighted controls. Performance of the late-blind subjects was somewhat worse than that of the early-blind subjects but substantially better than that of the sighted subjects. The lack of a significant difference between the late-blind subjects and sighted subjects is probably attributable to the higher variability among the late-blind subjects and their smaller number. These results are consistent with earlier findings that the blind are better than the sighted at recognition of Braille-like dot patterns (Foulke \& Warm, 1967). The use

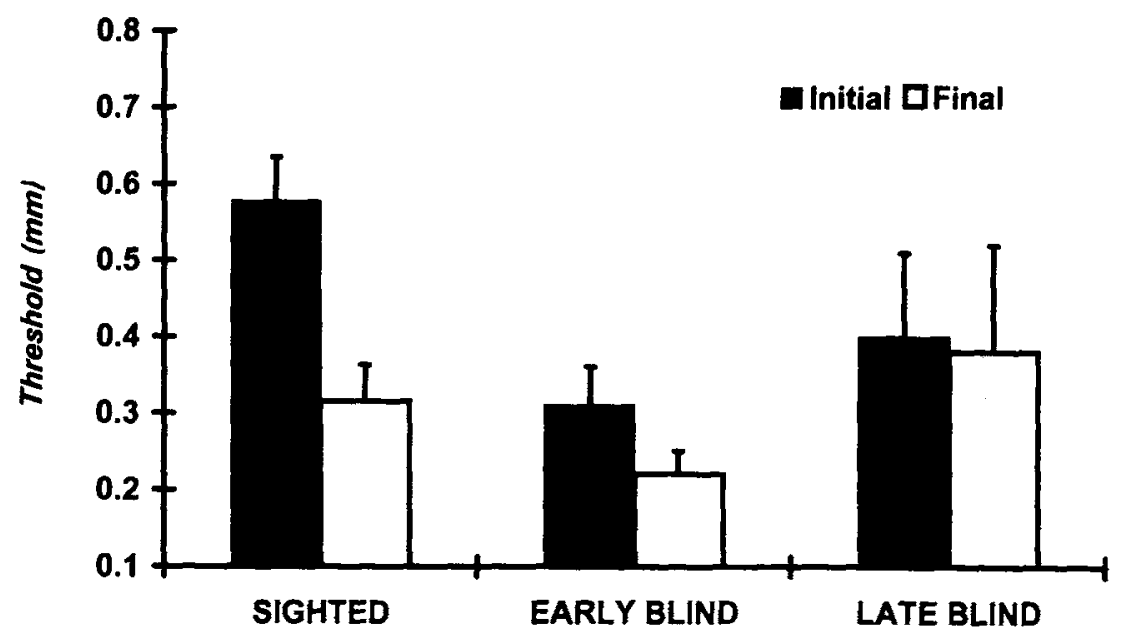

Figure 2. Dot pattern discrimination thresholds on the dominant hand. Bars $=S E M$ s. 
of Braille-like stimuli and the requirement for a perceptual judgment based on the details of dot position (i.e., texturaltype cues similar to those used in reading Braille) probably account for the initial advantage of the blind subjects.

Although the dot spacings we used were comparable to those of Braille, our dots were about one third of the diameter of typical Braille dots (about $1 \mathrm{~mm}$ ), and the extent of central dot offset in the comparison stimuli ranged down to an order of magnitude smaller than the minimal spatial distances in Braille (edge-to-edge spacing of about $1 \mathrm{~mm}$ between dots in adjacent cells). Moreover, the subjects performed the dot pattern discrimination task in a "static" mode (i.e., the fingerpad was stationary during contact), whereas Braille is read in an active scanning mode. Finally, our task called for a different kind of perceptual judgment than Braille reading. Both tasks depend on sensing and encoding textural cues related to dot spacing, but Braille additionally involves more complex perceptual and linguistic processing. These factors could well have interfered with transfer of perceptual learning from Braille to our discrimination task; but they did not, consistent with the generalization found between different Optacon tasks and Braille reading (Craig, 1988). The limits of perceptual learning due to everyday experience thus appear to be less narrow than those usually observed in laboratory experiments (Gilbert, 1994; Sagi \& Tanne, 1994; Sathian \& Zangaladze, 1997). Further work is required to confirm this possibility and to investigate whether it relates to the duration of prior perceptual experience or to its diversity. Whether carryover would operate in the reverse direction from that observed here-that is, from dot pattern discrimination (after practice) to Braille reading-is also currently unknown.

The fact that the sighted subjects achieved thresholds comparable to those of the blind subjects by three or four sessions confirms our earlier finding that perceptual learning with this task is rapid (Sathian \& Zangaladze, 1998). This is in agreement with results from use of the Optacon, in which experienced sighted subjects did as well as blind users of the device (Craig, 1988). Similar findings have been reported using the Tadoma method, in which deaf-blind individuals perceive speech tactually by placing a hand on the face of the speaker and monitoring changes in the face and neck associated with speech output (Reed, Rabinowitz, Durlach, \& Braida, 1985). With practice, normal subjects could perform as well as a deafblind person with years of experience with Tadoma (Reed, Doherty, Braida, \& Durlach, 1982; Reed, Rubin, Braida, $\&$ Durlach, 1978). These findings and ours support Hollins's (1989) contention that the blind do not have a truly supernormal tactile sense but rather come to acquire greater proficiency with nonvisual modalities due to high-level processes such as learning and attention. This is consonant with Gibson's (1969) view that selective attention and fine-tuning of active exploration are critical to perceptual learning. That learning effects were smaller in blind subjects suggests that their initial performance (and also the final performance of sighted subjects) was close to absolute limits.

The similar performance on both hands of blind subjects is consistent with the fact that tactile asymmetries across a variety of tasks are either small or nonexistent (Summers \& Lederman, 1990) and may also reflect the fact that the blind commonly learn to read Braille bimanually (Mousty \& Bertelson, 1985). Another possibility is that the effects of Braille-reading experience with predominantly one hand are manifest bilaterally, due to the facility with which tactile learning effects appear to transfer between hands (Sathian \& Zangaladze, 1997, 1998).

\section{EXPERIMENT 2 Hyperacute Discrimination of Grating Ridge Width}

The results of Experiment 1 indicate that perceptual learning due to Braille-reading experience does result in significantly better performance on a tactile hyperacuity task employing Braille-like dot patterns, despite some differences between the task used and Braille reading. Experiment 2 tested whether this tactile superiority of the blind would generalize to discrimination of periodic gratings differing in their spatial dimensions, another textural task for which performance is in the hyperacute range (Morley et al., 1983; Sathian \& Zangaladze, 1997). Grating discrimination is typically tested with the subject's fingerpad sweeping across stationary gratings, a movement similar to that used in reading Braille. Gratings, however, are quite unlike Braille dot patterns. Moreover, discrimination of gratings varying in their spatial dimensions is a completely different task than recognition of Braille characters. The blind have experience not only with reading Braille but also presumably with judging textures of various objects and surfaces. If they were to outperform sighted controls on grating discrimination, it would imply that their experience with such tactile textural tasks does confer a generalizable advantage, possibly related to a common means of information pick-up (active scanning). Although no difference was reported between sighted and blind subjects in their ability to discriminate the smoothness of various grades of sandpaper (Heller, $1989 \mathrm{~b}$ ), it is possible that use of more precisely specified stimuli to measure a discrimination threshold might reveal differences that were not found in this earlier study, which did not formally assess discriminability.

Periodic gratings consist of alternating ridges and grooves (Figure 1B) whose widths can be specified to an accuracy of $0.01 \mathrm{~mm}$ (Sathian, 1989). Perceived grating roughness increases as groove width $(\mathrm{GW})$ increases or ridge width (RW) decreases, the effect of RW being smaller (Lederman \& Taylor, 1972; Sathian, Goodwin, John, \& Darian-Smith, 1989). Neurophysiologic studies in monkeys indicate that changes in GW and RW are re- 
flected in changes in responses of all three major mechanoreceptive afferent classes: SAIs, RAs, and Pacinians (Sathian et al., 1989). Normal subjects have higher thresholds for discrimination of RW than of GW (Sathian \& Zangaladze, 1997). We therefore chose to use gratings differing in RW, in order to maximize the likelihood of finding any possible superiority in the blind.

\section{Method}

A series of steel-backed plastic gratings with constant GW (approximately $1.0 \mathrm{~mm}$ ) and varying RW were used as stimuli (Figure 1B). As previously described (Sathian \& Zangaladze, 1997), the subject compared two gratings presented on adjacent magnetic holding wells: a standard grating with RW of $1.95 \mathrm{~mm}$, and a comparison grating whose RW was smaller than that of the standard by $0.05-1.45 \mathrm{~mm}(\triangle \mathrm{RW})$. Full details of grating spatial parameters and of grating preparation can be found in a previous report (Sathian \& Zangaladze, 1997). The subject scanned the gratings with the index fingerpad, with no constraints on number of scans, movement speed, or contact force. On the right was the standard grating; on the left was either a copy of the standard or a comparison grating that was constant within a block of 24 trials and varied between blocks. The subject had to state whether the gratings felt the same or different. A session lasted $30-45 \mathrm{~min}$ and typically included four to six blocks. As in Experiment 1, both hands of the blind subjects were tested, with the dominant hand tested first; only the dominant hand was tested in the sighted controls.

Comparison gratings were presented in a sequence of increasing difficulty. The initial comparison grating for each subject was chosen on the basis of a few practice trials (for the first session) or previous performance (for subsequent sessions). Since criterion shift with this task could affect threshold determination, the bias-free separation index, $d^{\prime}$, was computed as the difference between $z$ scores for hits and false alarms (Macmillan \& Creelman, 1991). The difference limen (DL) was taken as the $\Delta \mathrm{RW}$ yielding a $d^{\prime}$ of 1.35 , which corresponds to $75 \%$ correct, unbiased performance.

Substantial perceptual learning occurs with this task as well, the DL declining by about $50 \%$ after several sessions (Sathian \& Zangaladze, 1997). We therefore attempted to test the subjects on four sessions with this task. ${ }^{4}$ The DL in the first session was taken as the initial DL. Since the DLs did not differ significantly between the
Table 2

Data for the Sighted Subjects on Discrimination of Grating Ridge Width

\begin{tabular}{lcccc}
\hline & & & \multicolumn{2}{c}{ Difference Limen (mm) } \\
\cline { 4 - 5 } Subject & Age (years) & Gender & Initial & Final \\
\hline S20 & 22 & F & 0.12 & 0.19 \\
S21 & 46 & M & 1.45 & 0.90 \\
S22 & 21 & F & 0.23 & 0.14 \\
S26 & 54 & F & 0.67 & 1.02 \\
S27 & 53 & F & 1.13 & 1.45 \\
S28 & 21 & F & 0.39 & 0.37 \\
S29 & 22 & M & 0.95 & 0.64 \\
S30 & 53 & F & 0.45 & 0.67 \\
S31 & 44 & F & 0.27 & 0.16 \\
S32 & 29 & F & 0.19 & 0.22 \\
S33 & 19 & F & & 0.19 \\
S34 & 48 & F & 0.65 & 0.60 \\
S35 & 25 & F & 0.62 & 0.20 \\
M & 35.2 & & 0.59 & 0.52 \\
SEM & 4.0 & & 0.12 & 0.11 \\
\hline
\end{tabular}

last two sessions, ${ }^{5}$ a final DL was obtained on the basis of the raw performance data pooled over these two sessions.

\section{Results}

The DLs for discrimination of grating RW are shown in Table 2 for the sighted subjects and in Table 4 for the blind subjects (RW columns). Figure 3 illustrates the performance data by group for the dominant hand. It suggests a trend for the blind subjects to perform better than the sighted, although there was considerable variability within each group (note the relatively high standard errors). A one-way ANOVA with initial DL on the dominant hand as the dependent variable revealed no significant effect of group [control, early blind, and late blind; $F(2,30)=$ $0.32, p=.73]$. A further repeated measures ANOVA on initial DLs of the blind subjects showed no significant (between-subjects) effect of subgroup [early vs. late blind;

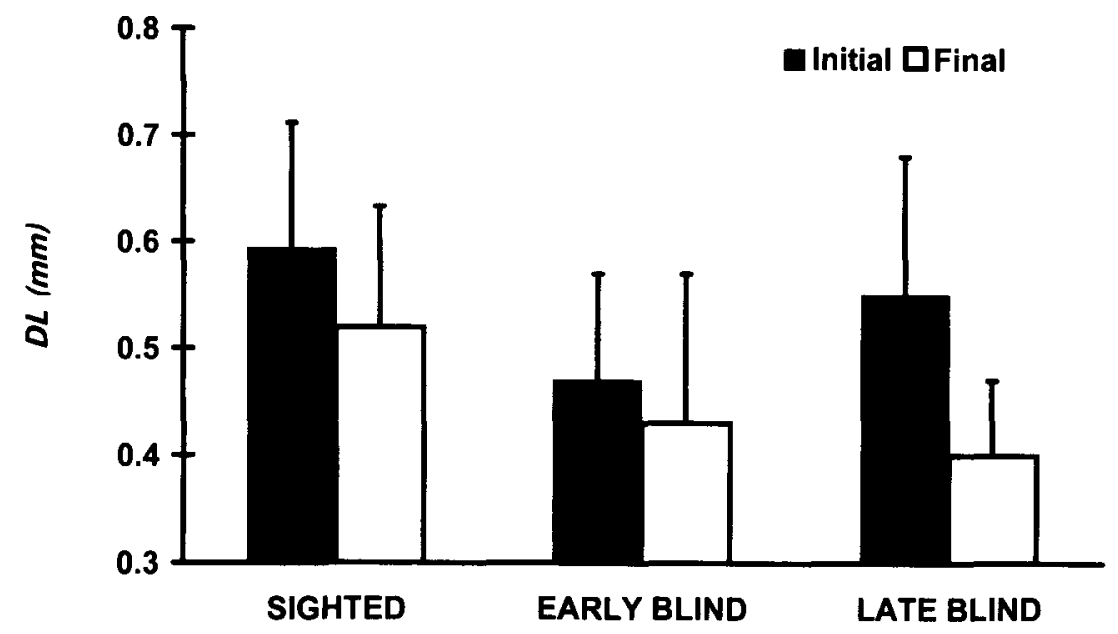

Figure 3. Difference limens (DLs) for discrimination of grating RW on the dominant hand. Bars $=S E M$ s. 
$F(1,15)=0.57, p=.46]$, no significant (within-subjects) effect of hand [dominant vs. nondominant; $F(1,15)=$ $2.58, p=.13]$, and no significant interaction $[F(1,15)=$ $1.02, p=.33$ ]. Similar results were obtained for final DLs: There was no significant effect of group on performance with the dominant hand on a one-way ANOVA $[F(2,29)=0.28, p=.76]$; on the subsequent repeated measures ANOVA for the blind subjects, there was no significant effect of subgroup $[F(1,14)=0.39, p=.54]$, hand $[F(1,14)=2.06, p=.17]$, or interaction $[F(1,14)=$ $0.28, p=.6]$.

\section{Discussion}

The absence of a significant difference between the blind and sighted subjects in this experiment is consistent with the findings of Heller (1989b) in his study of discrimination of sandpaper texture. The experience of the blind with surface textures could have been expected to result in superior grating discrimination. However, perhaps gratings are too different from textures routinely encountered in everyday life, or the difference between the blind and sighted in their tactile textural experience may be insufficient to result in a measurable difference in acuity. It is also possible that the relatively high withingroup variances in the grating RW discrimination task could have masked a difference between groups.

In Experiment 1, tactile hyperacuity was found to be significantly better in the blind subjects than in the sighted subjects. This was attributed to blind subjects' Braillereading experience, implying generalizability of perceptual learning to a rather different task, albeit with similar stimuli. Since, in Experiment 2, superior performance was not found on another hyperacuity task, grating discrimination, it appears that such generalizability has finite limits and that the blind do not have an overall increase in their tactile sensitivity. The results of this experiment also imply that the motoric similarity of grating discrimination to reading Braille is not sufficient to provide blind subjects with a significant advantage.

\section{EXPERIMENT 3 Spatial-Acuity-Dependent Discrimination of Grating Orientation}

Experiment 3 provided an opportunity to test the conclusion derived from the previous two experimentsnamely, that the blind do not show a general superiority of tactile acuity. We used discrimination of grating orientation (Figure $1 \mathrm{C}$ ) in this experiment. This ability depends on grating resolution and is a monotonically increasing function of GW up to $3 \mathrm{~mm}$ (Johnson \& Phillips, 1981; van Boven \& Johnson, 1994a). The GW at the threshold of orientation discrimination is a reliable index of tactile spatial acuity in particular (Johnson \& Phillips, 1981; van Boven \& Johnson, 1994a) and of tactile sensitivity in general (van Boven \& Johnson, 1994b). The firing rates of mechanoreceptive afferents innervating the fingerpad increase with GW, the responses of SAI afferents show- ing the clearest representation of grating spatial profiles (Phillips \& Johnson, 1981).

This task is of interest for another reason. During psychophysical studies of grating orientation discrimination in our laboratory, sighted subjects reported that they mentally visualized the tactile stimulus. It has previously been suggested that visual imagery is involved in certain tactile tasks but not others. For instance, when sighted subjects explored objects haptically with vision excluded, instructions to sort according to similarity of visual images led to emphasis of cues such as object shape (Klatzky, Lederman, \& Reed, 1987). Without such instruction, object properties such as texture dominated. The terms macrogeometric and microgeometric have been used to distinguish two classes of object properties, shape belonging in the former class and texture in the latter (Roland \& Mortensen, 1987). Operationally, microgeometric properties can be defined as those on a spatial scale under $3 \mathrm{~mm}$, since responses to adjacent stimuli are independent when their separation is above this limit but not below it (Phillips \& Johnson, 1981). Distinct cerebral cortical areas appear to mediate tactile processing of microgeometric versus macrogeometric properties (Randolph \& Semmes, 1974; Roland, 1987). The stimuli used in Experiments 1 and 2 and the spatial dimensions of the gratings used in the present experiment varied in the microgeometric range, but changes in orientation are on the macrogeometric scale.

In a functional neuroimaging study in sighted individuals during discrimination of grating orientation, we found significant activation in an area of parieto-occipital cortex (Sathian, Zangaladze, Hoffman, \& Grafton, 1997), relative to a control microgeometric task in which subjects discriminated grating spatial dimensions. Interfering with the function of this area using TMS disrupted tactile discrimination of grating orientation but not spatial frequency (Zangaladze, Epstein, Grafton, \& Sathian, 1999). This area is also active during visual discrimination of grating orientation (Sergent, Ohta, \& MacDonald, 1992) and may correspond to a visual area in the parieto-occipital fissure of the macaque brain, known as area V6 or PO, where a large proportion of neurons are orientation-selective (Galletti, Battaglini, \& Fattori, 1991). We proposed that the parieto-occipital cortical activation occurred in a "topdown" manner to enable comparison of tactile input with a stored visual representation; alternatively, this area could be multimodal (Sathian et al., 1997). If, indeed, visual imagery is crucial for tactile discrimination of orientation, blind subjects should be impaired at discriminating grating orientation and the early blind might be especially impaired due to minimal or no visual experience. A competing a priori prediction is that experience with haptic spatial exploration, during the course of which oriented edges are probably frequently encountered, might result in normal or even supernormal performance in the blind.

\section{Method}

Tactile spatial acuity was determined as described previously (Sathian \& Zangaladze, 1996; van Boven \& Johnson, 1994a), using 
a set of eight commercially available plastic domes (JVP Domes; Stoelting, Wood Dale, IL) whose faces were cut with gratings of equal RW and GW (Figure 1C). Values of GW were 3, 2, 1.5, 1.2, $1,0.75,0.5$, and $0.35 \mathrm{~mm}$. The subject's hand was supine, with the index finger immobilized. The gratings were manually applied to the index fingerpad for approximately $1 \mathrm{sec}$, with the ridges oriented either along or across the fingerpad. Following each application, the subject verbally reported grating orientation.

Testing was conducted in two sessions. The first session began with either a 3-mm or a 2-mm grating and continued with successively smaller gratings, until performance fell below $75 \%$ correct on two successive domes. Each dome was presented for a block of trials, usually 50 . Each block contained an equal number of the two grating orientations, arranged in a pseudorandom sequence. Both hands were tested; the dominant hand was always tested first. The second session was identical to the first except that each subject's performance during the first session was used as a guide for choosing an appropriate sequence of domes for the second. Although perceptual learning does occur in this task, learning effects are of relatively small magnitude (Sathian \& Zangaladze, 1997; van Boven \& Johnson, 1994a). There was no significant threshold difference between the first session and the second session in blind and sighted groups on either hand. ${ }^{6}$ The raw performance scores for each dome were therefore averaged across the two sessions and used to compute the discrimination threshold as the GW corresponding to $75 \%$ correct performance. When a subject failed to achieve $75 \%$ correct with the widest GW of $3 \mathrm{~mm}$, this was taken as the threshold for statistical purposes.

\section{Results}

Table 3 shows the thresholds for grating orientation discrimination for the sighted subjects. The corresponding values for the blind subjects are shown in Table 4 (OR columns). As Figure 4 illustrates, the early-blind subjects had a higher mean threshold than the sighted subjects, and the late-blind subjects had a somewhat higher threshold with their nondominant hand than with the dominant hand. However, a repeated measures ANOVA with discrimination threshold as the dependent variable showed that neither the main (between-subjects) effect of group [control, early blind, and late blind; $F(2,44)=1.14, p=.33$ ] nor the main (within-subjects) effect of hand [dominant vs. nondominant; $F(1,44)=1.31, p=.26$ ] was significant. The interaction between these two independent variables was also not significant $[F(2,44)=2.1, p=.14]$.

\section{Discussion}

There was no significant difference in grating orientation discrimination threshold between groups, reinforcing the finding from Experiment 2 that the blind do not have generally superior tactile sensitivity. This result also implies that, whatever the role of visual imagery may be in sighted subjects, it is not imperative for optimal performance. The long-standing haptic experience of the blind could compensate for deficient visual imagery. Alternatively, if the area of parieto-occipital cortex activated in our functional imaging study is multimodal and equivalently activated by tactile and visual inputs, blindness may be of no consequence for this activation and for discrimination of grating orientation. The blind subjects' experience with haptic exploration of their environment
Table 3

Data for the Sighted Subjects on Grating Orientation Discrimination

\begin{tabular}{ccccc}
\hline & & & \multicolumn{2}{c}{ Threshold (mm) } \\
\cline { 3 - 5 } Subject & Age (years) & Gender & Dom & Nondom \\
\hline S1 & 47 & F & 0.92 & 0.88 \\
S2 & 51 & F & 1.23 & 0.73 \\
S3 & 42 & F & 1.68 & 1.73 \\
S4 & 65 & M & 1.58 & 1.66 \\
S5 & 45 & M & 0.74 & 0.72 \\
S6 & 51 & F & 0.96 & 0.98 \\
S7 & 49 & F & 1.39 & 1.48 \\
S8 & 75 & F & 1.94 & 1.88 \\
S9 & 53 & M & 0.86 & 0.71 \\
S10 & 44 & F & 0.64 & 0.96 \\
S11 & 56 & F & 1.91 & 2.42 \\
S12 & 53 & F & 0.71 & 0.94 \\
S13 & 39 & M & 1.14 & 0.95 \\
S14 & 31 & M & 1.18 & 0.96 \\
S15 & 23 & F & 0.97 & 1.13 \\
S16 & 25 & M & 0.90 & 0.64 \\
S17 & 30 & F & 0.70 & 0.60 \\
S18 & 22 & F & 0.90 & 0.96 \\
S19 & 19 & M & 0.92 & 0.55 \\
S20 & 22 & F & 0.93 & 0.80 \\
S21 & 46 & M & 2.96 & 1.75 \\
S22 & 21 & F & 1.09 & 1.84 \\
S23 & 44 & F & 1.97 & 1.07 \\
S24 & 22 & F & 1.65 & 1.54 \\
S25 & 20 & M & 2.09 & 1.84 \\
M & 39.8 & & 1.28 & 1.19 \\
SEM & 3.1 & & 0.11 & 0.10 \\
\hline
\end{tabular}

Note-Dom, dominant hand; Nondom, nondominant hand.

did not translate into better-than-normal performance in this experiment, although we have no way of assessing the frequency with which they typically encounter oriented edges or make haptic judgments of orientation.

Despite the lack of significant interaction between subject group and hand on ANOVAs, we were interested in the suggestion of asymmetry in the late-blind subgroup. The mean threshold for this subgroup was $17 \%$ higher on the nondominant hand than on the dominant hand (among subjects tested on both hands); this difference was significant by a paired $t$ test $(p=.04)$. Corresponding comparisons showed no significant difference between hands either for the early-blind subgroup ( $p=$ .38 ) or for the sighted controls ( $p=.27$ ), replicating our earlier findings in sighted subjects (Sathian \& Zangaladze, 1996). This raises the intriguing possibility that, in the late blind, use of the dominant hand for spatial exploration may preserve dominant hemispheric activity of multimodal regions involved in this task, whereas lack of use of the nondominant hand could result in waning of the ability to recruit such activity in the corresponding region(s) of the nondominant hemisphere, according to Hebbian principles. If this is true, it would support the idea that haptic practice could be responsible for the normal performance of the blind subjects. The asymmetry also violates the principle that tactile learning effects appear to transfer effectively between hands (Sathian \& Zan- 
Table 4

Data for the Blind Subjects, Grouped Separately for Early and Late Blind

\begin{tabular}{|c|c|c|c|c|c|c|c|c|c|c|c|c|}
\hline \multirow[b]{3}{*}{ Subject } & \multirow[b]{3}{*}{ Age } & \multirow[b]{3}{*}{ Gender } & \multicolumn{4}{|c|}{$\mathrm{DP}$} & \multicolumn{4}{|c|}{ RW } & \multirow{2}{*}{\multicolumn{2}{|c|}{ OR }} \\
\hline & & & Initial & Final & & & Initial & Final & Initial & & & \\
\hline & & & Dom & Dom & Nondom & Nondom & Dom & Dom & Nondom & Nondom & Dom & Nondom \\
\hline \multicolumn{13}{|l|}{ Early Blind } \\
\hline $\mathrm{Bl}$ & 23 & M & 0.52 & & 0.40 & & 0.14 & 0.18 & 0.15 & 0.05 & 1.88 & 2.40 \\
\hline $\mathrm{B} 2$ & 42 & $\mathrm{M}$ & 0.25 & 0.13 & 0.17 & 0.19 & 0.77 & 0.21 & 0.41 & 0.30 & 1.22 & 1.07 \\
\hline B3 & 23 & $\mathrm{M}$ & 0.23 & & & & 0.24 & 0.29 & & & 0.98 & 1.48 \\
\hline B4 & 46 & M & 0.38 & 0.25 & 0.35 & & 0.82 & 1.21 & 0.65 & 0.74 & 3.00 & 3.00 \\
\hline B5 & 27 & $\mathrm{M}$ & 0.73 & 0.28 & 0.26 & 0.24 & 0.32 & 0.21 & 0.21 & 0.13 & 0.72 & 0.96 \\
\hline B6 & 18 & $\mathrm{~F}$ & 0.28 & 0.12 & 0.17 & 0.10 & 0.25 & 0.18 & 0.20 & 0.25 & 1.09 & 0.81 \\
\hline B7 & 48 & $\mathrm{~F}$ & & & & & & & & & 1.27 & 2.40 \\
\hline B8 & 42 & $\mathrm{M}$ & & & & & 0.70 & & & & 3.00 & 3.00 \\
\hline B9 & 70 & $\mathrm{~F}$ & 0.38 & 0.43 & 0.55 & 0.57 & & & 0.45 & 0.49 & 2.39 & 1.55 \\
\hline B 10 & 26 & $\mathrm{M}$ & 0.10 & & 0.17 & & 0.94 & 0.31 & 0.64 & 0.66 & 1.23 & 1.50 \\
\hline B 11 & 50 & $F$ & 0.10 & 0.25 & 0.33 & 0.10 & 0.18 & 0.20 & 0.59 & 0.11 & 0.89 & 0.84 \\
\hline B 12 & 37 & $\mathrm{~F}$ & 0.22 & 0.14 & 0.26 & 0.22 & 0.17 & 0.23 & 0.12 & 0.16 & 0.71 & 0.81 \\
\hline B 13 & 43 & $\mathrm{M}$ & 0.15 & 0.34 & 0.50 & 0.35 & 0.95 & 1.45 & 1.19 & & 1.18 & 1.71 \\
\hline B 14 & 44 & $\mathrm{~F}$ & 0.43 & 0.22 & 0.70 & 0.18 & 0.17 & 0.28 & 0.17 & 0.25 & 1.04 & 0.73 \\
\hline B15 & 49 & $F$ & & & & & & & & & 1.39 & 1.34 \\
\hline$M$ & 39.2 & & 0.31 & 0.24 & 0.35 & 0.24 & 0.47 & 0.43 & 0.43 & 0.31 & 1.47 & 1.57 \\
\hline$S E M$ & 3.6 & & 0.05 & 0.03 & 0.05 & 0.05 & 0.10 & 0.14 & 0.10 & 0.08 & 0.20 & 0.20 \\
\hline \multicolumn{13}{|l|}{ Late Blind } \\
\hline B 16 & 38 & M & & & & & 0.20 & & & & 0.93 & \\
\hline B17 & 39 & $\mathrm{~F}$ & 0.10 & 0.10 & & & 0.20 & 0.18 & & & 0.71 & \\
\hline B 18 & 43 & $\mathrm{~F}$ & 0.35 & & 0.29 & 0.30 & 0.53 & 0.67 & 0.57 & 0.53 & 1.14 & 1.39 \\
\hline B19 & 37 & $F$ & 0.19 & 0.13 & 0.28 & 0.25 & 0.67 & 0.28 & 0.49 & 0.25 & 1.16 & 1.07 \\
\hline $\mathrm{B} 20$ & 47 & M & 0.65 & 0.28 & 0.63 & 0.43 & 0.31 & 0.34 & 0.58 & 0.11 & 2.07 & 2.43 \\
\hline B21 & 48 & M & 1.00 & 1.00 & 1.00 & & 0.53 & 0.56 & 0.35 & 0.61 & 1.46 & 2.04 \\
\hline $\mathrm{B} 22$ & 43 & M & 0.33 & & 0.13 & 0.33 & 0.24 & 0.31 & 0.20 & 0.15 & 0.75 & 0.95 \\
\hline B23 & 52 & $\mathrm{~F}$ & 0.43 & 0.59 & 0.55 & & 1.45 & 0.62 & 0.87 & 0.55 & 1.14 & 1.20 \\
\hline B24 & 36 & $M$ & 0.12 & 0.18 & 0.22 & 0.10 & 0.83 & 0.25 & 0.33 & 0.22 & 1.08 & 1.19 \\
\hline$M$ & 42.6 & & 0.40 & 0.38 & 0.44 & 0.28 & 0.55 & 0.40 & 0.48 & 0.35 & 1.16 & 1.47 \\
\hline$S E M$ & 1.9 & & 0.11 & 0.14 & 0.11 & 0.05 & 0.13 & 0.07 & 0.08 & 0.08 & 0.14 & 0.21 \\
\hline \multicolumn{13}{|l|}{ All Blind } \\
\hline$M$ & 40.5 & & 0.35 & 0.29 & 0.39 & 0.26 & 0.51 & 0.42 & 0.45 & 0.33 & 1.35 & 1.54 \\
\hline$S E M$ & 2.3 & & 0.05 & 0.06 & 0.05 & 0.04 & 0.08 & 0.08 & 0.07 & 0.05 & 0.13 & 0.15 \\
\hline
\end{tabular}

Note-DP, dot pattern discrimination threshold; RW, difference limen for grating ridge width discrimination; OR, grating orientation discrimination threshold; Dom, dominant hand; Nondom, nondominant hand.

galadze, 1997, 1998). Clearly, further work is required to fully assess these issues.

\section{GENERAL DISCUSSION}

Blind Braille readers outperformed sighted subjects on a tactile hyperacuity task calling for a perceptual judgment based on the position of a given dot in a Braille-like dot pattern. The equivalent practiced performance of both subject groups on this task is consistent with earlier observations that people deprived of one or more senses do not develop truly supernormal sensitivities of the remaining ones but rather learn to use them proficiently for purposes other than those for which they are normally used (Craig, 1988; Hollins, 1989; Reed et al., 1982; Reed et al., 1978).

Although the dot pattern task differs from Braille reading in some key aspects, it is sufficiently similar to Braille to conclude that the Braille-reading experience of the blind probably accounts for their superior performance, allowing for perceptual learning effects that are not as highly task-specific as in most laboratory studies (Gilbert, 1994; Sagi \& Tanne, 1994; Sathian \& Zangaladze, 1997). The blind performed at comparable levels to the sighted on two other tasks using gratings. One of these tasks involved hyperacute discrimination between gratings varying in RW. The other was a test of spatial resolving acuity as indexed by the minimal GW permitting reliable discrimination of grating orientation. These findings indicate that transfer of perceptual learning effects due to tactile experience has finite limits. Moreover, the restriction of tactile superiority in the blind to the dot pattern task implies that expansion of topographic representations in somatosensory cortex in blindness (PascualLeone \& Torres, 1993; Pascual-Leone et al., 1995) and in various other situations (Merzenich \& Jenkins, 1993) is not necessarily associated with a general improvement in tactile sensitivity or acuity.

Functional neuroimaging studies reveal that visual cortical areas of blind (but not sighted) subjects are active during various tactile tasks using Braille patterns as stimuli (Büchel, Price, Frackowiak, \& Friston, 1998; Sa- 


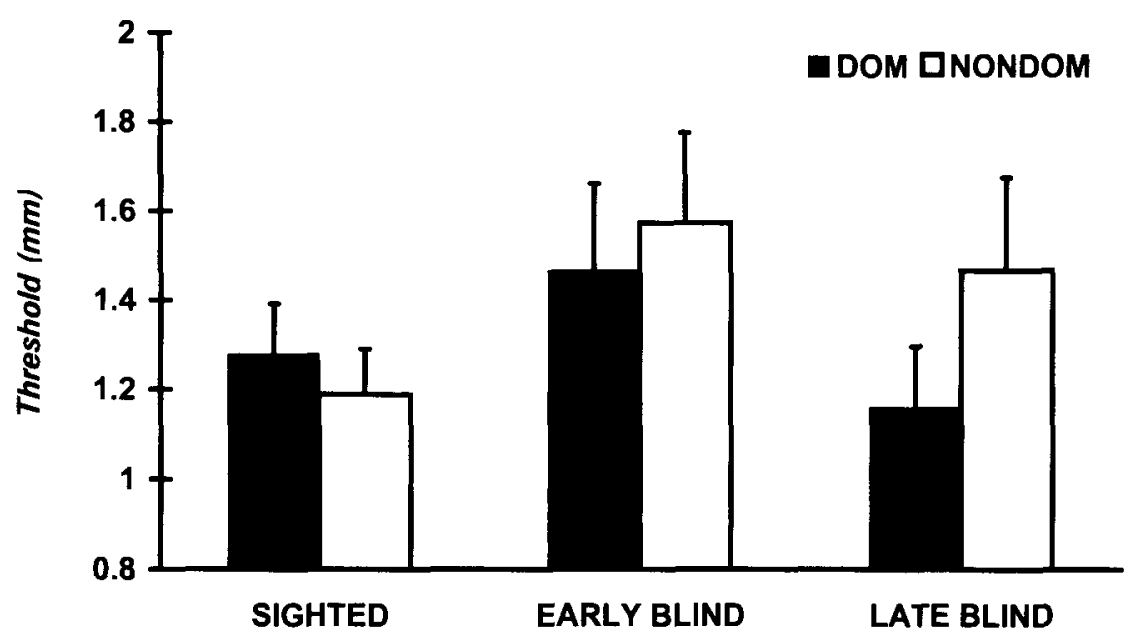

Figure 4. Grating orientation discrimination thresholds. Dom, dominant hand; Nondom, nondominant hand. Bars $=S E M$ s.

dato et al., 1998; Sadato et al., 1996). Sighted subjects show activity in parietal opercular cortex (where the second somatosensory cortex, or SII, is located), whereas this region is deactivated in the blind (Sadato et al., 1998). Interference with occipital cortical function by TMS disrupts tactile pattern-recognition performance in blind subjects but not in sighted subjects (Cohen et al., 1997). These findings imply that visual cortical areas are involved in processing tactile information in the blind. One group reported that activity in primary visual cortex during a Braille task is seen only in late-blind subjects but not in early-blind subjects (Büchel et al., 1998); however, another group found no such difference (Sadato et al., 1998). It is interesting that there were no clear differences between early- and late-blind subjects on any of our tasks. This suggests that any differences between these two subgroups of blind subjects in the extent to which visual cortical regions are involved in somatosensory processing may not necessarily correlate with performance, assuming that our results are not due to Type II statistical error or to the fact that some of our late-blind subjects did have some degree of visual impairment in early life. Performance measures such as Braille-reading speed do not differ clearly between early-and late-blind subjects (Mousty $\&$ Bertelson, 1985). Moreover, the role of visual cortical activity could differ between high-level tasks, such as Braille reading, that require more complex sensory and cognitive processing than purely sensory discriminations such as those studied in the present report. Potential differences between early and late blind are obviously of great interest and deserve further study. The duration of blindness is another complicating factor; however, all of our subjects had been blind for at least 12 years.

We believe that our work provides further impetus for careful study of tactile perception following visual de- privation using psychophysical methods coupled with functional neuroimaging and neurophysiologic studies.

\section{REFERENCES}

AdAMS, R. D., \& Victor, M. (1993). Principles of neurology (5th ed.). New York: McGraw-Hill.

BaILES, S. M., \& LAmberT, R. M. (1986). Cognitive aspects of haptic form recognition by blind and sighted subjects. British Journal of $P$ sychology, 77, 451-458.

Büchel, C., Price, C., Frackowiak, R. S. J., \& Friston, K. (1998). Different activation patterns in the visual cortex of late and congenitally blind subjects. Brain, 121, 409-419.

Cohen, L. G., Celnik, P., Pascual-Leone, A., Corwell, B., Faiz, L., Dambrosia, J., Honda, M., Sadato, N., Gerloff, C., Catala, M. D., \& HalletT, M. (1997). Functional relevance of cross-modal plasticity in blind humans. Nature, 389, 180-183.

CraiG, J.C. (1988). The role of experience in tactual pattern perception: A preliminary report. International Journal of Rehabilitation Research, 11, 167-183.

D'Angiulli, A., Kennedy, J. M., \& Heller, M. A. (1998). Blind children recognizing tactile pictures respond like sighted children given guidance in exploration. Scandinavian Journal of Psychology, 39, $187-190$.

Davidson, P. W. (1972). Haptic judgments of curvature by blind and sighted humans. Journal of Experimental Psychology, 93, 43-55.

FoULKE, E., \& WARM, J. S. (1967). Effects of complexity and redundancy on the tactual recognition of metric figures. Perceptual \& Motor Skills, 25, 177-187.

Galletti, C., Battaglini, P. P., \& Fattori, P. (1991). Functional properties of neurons in the anterior bank of the parieto-occipital sulcus of the macaque monkey. European Journal of Neuroscience, 3 , 452-461.

GiBson, E. J. (1969). Principles of perceptual learning and development. New York: Meredith.

GilberT, C. D. (1994). Early perceptual learning. Proceedings of the National Academy of Sciences, 91, 1195-1197.

Grant, A. C., Thiagarajah, M., Chevalier, S. J., \& Sathian, K. (1997). Tactile perception in blind Braille readers. Society for Neuroscience Abstracts, 23, 98.14.

Grant, A. C., Zangaladze, A., Thiagarajah, M. C., \& Sathian, K. (1999). Tactile perception in developmental dyslexia: A psychophysical study using gratings. Neuropsychologia, 37, 1201-1211. 
GRIESBaCH, H. (1899). Vergleichende Untersuchungen über die Sinnesschärfe Blinder und Sehender [Comparative studies of perceptual acuity in the blind and the sighted]. Pflügers Archiv, 74, 577-638.

HELlER, M. A. (1989a). Picture and pattern perception in the sighted and the blind: The advantage of the late blind. Perception, 18, 379-389.

Heller, M. A. (1989b). Texture perception in sighted and blind observers. Perception \& Psychophysics, 45, 49-54.

Heller, M. A., Calcaterra, J. A., Tyler, L. A., \& Burson, L. L. (1996). Production and interpretation of perspective drawings by blind and sighted people. Perception, 25, 321-334.

Heller, M. A., Kennedy, J. M., \& Joyner, T. D. (1995). Production and interpretation of pictures of houses by blind people. Perception, 24, 1049-1058.

Hol.ins, M. (1989). Understanding blindness. Hillsdale, NJ: Erlbaum.

HUBEL, D. H., \& WiESEL, T. N. (1977). Ferrier Lecture: Functional architecture of macaque monkey visual cortex. Proceedings of the Royal Society of London: Series B, 198, 1-59.

Johansson, R. S., \& VAllbo, A. B. (1979). Tactile sensibility in the human hand: Relative and absolute densities of four types of mechanoreceptive units in glabrous skin. Journal of Physiology, 286, 283-300.

Johnson, K. O., \& LAMB, G. D. (1981). Neural mechanisms of spatial tactile discrimination: Neural patterns evoked by Braille-like dot patterns in the monkey. Journal of Physiology, 310, 117-144.

Johnson, K. O., \& Phillips, J. R. (1981). Tactile spatial resolution. I. Two-point discrimination, gap detection, grating resolution and letter recognition. Journal of Neurophysiology, 46, 1177-1191.

KenNedy, J. M. (1997). How the blind draw. Scientific American, 276 , 60-65.

Klatzky, R. L., Lederman, S., \& Reed, C. (1987). There's more to touch than meets the eye: The salience of object attributes for haptics with and without vision. Journal of Experimental Psychology: General, 116, 356-369.

Krueger, L. E. (1970). David Katz's Der Aufbau der Tastwelt (The world of touch): A synopsis. Perception \& Psychophysics, 7, 337-341.

Lederman, S. J., Klatzky, R. L., Chataway, C., \& Summers, C. D. (1990). Visual mediation and the haptic recognition of two-dimensional pictures of common objects. Perception \& Psychophysics, 47, 54-64.

Lederman, S. J., \& TAYLOR, M. M. (1972). Fingertip force, surface geometry, and the perception of roughness by active touch. Perception \& Psychophysics, 12, 401-408.

LoOMIS, J. M. (1979). An investigation of tactile hyperacuity. Sensory Processes, 3, 289-302.

Loomis, J. M. (1981). On the tangibility of letters and Braille. Perception \& Psychophysics, 29, 37-46.

Macmillan, N. A., \& Creelman, C. D. (1991). Detection theory: A user's guide. Cambridge: Cambridge University Press.

MEENES, M., \& Zigler, M. J. (1923). An experimental study of the perceptions roughness and smoothness. American Journal of Psychologv, 34, 542-549.

Merzenich, M. M., \& Jenkins, W. M. (1993). Reorganization of cortical representations of the hand following alterations of skin inputs induced by nerve injury, skin island transfers, and experience. Journal of Hand Therapy, 6, 89-104.

MILLAR, S. (1994). Understanding and representing space: Theory and evidence from studies with blind and sighted children. New York: Oxford University Press.

Morley, J. W., Goodwin, A. W., \& Darian-Smith, I. (1983). Tactile discrimination of gratings. Experimental Brain Research, 49, 291-299.

Morrongiello, B. A., Humphrey, K., Timney, B., Choi, J., \& Rocca, P. T. (1994). Tactual object exploration and recognition in blind and sighted children. Perception, 23, 833-848.

Mousty, P., \& Bertelson, P. (1985). A study of Braille reading: 1. Reading speed as a function of hand usage and context. Quarterly Journal of Experimental Psychology, 37A, 217-233.

Pascual-Leone, A., \& Torres, F. (1993). Plasticity of the sensorimotor cortex representation of the reading finger in Braille readers. Brain, 116, 39-52.

Pascual-Leone, A., Wasserman, E. M., Sadato, N., \& Hallett, M.
(1995). The role of reading activity on the modulation of motor cortical outputs to the reading hand in Braille readers. Annals of Neurology, 38, 910-915

Phillips, J. R., \& Johnson, K. O. (1981). Tactile spatial resolution: II. Neural representation of bars, edges and gratings in monkey primary afferents. Journal of Neurophysiology, 46, 1192-1203.

Phillips, J. R., Johnson, K. O., \& Browne, H. M. (1983). A comparison of visual and two modes of tactual letter resolution. Perception \& Psychophysics, 34, 243-249.

Raczkowski, D., Kalat, J. W., \& Nebes, R. (1974). Reliability and validity of some handedness questionnaire items. Neuropsychologia, 12, 43-47.

RANDOLPH, M., \& SEMmES, J. (1974). Behavioral consequences of selective subtotal ablations in the postcentral gyrus of Macaca mulatta. Brain Research, 70, 55-70.

ReEd, C. M., Doherty, M. J., Braida, L. D., \& Durlach, N. I. (1982). Analytic study of the Tadoma method: Further experiments with inexperienced observers. Journal of Speech \& Hearing Research, 25, 216-223.

ReEd, C. M., Rabinowitz, W. M., Durlach, N. I., \& Braida, L. D. (1985). Research on the Tadoma method of speech communication. Journal of the Acoustical Society of America, 77, 247-257.

ReEd, C. M., Rubin, S. I., Braida, L. D., \& Durlach, N. I. (1978). Analytic study of the Tadoma method: Discrimination ability of untrained observers. Journal of Speech \& Hearing Research, 21, 625-637.

RoLAND, P. E. (1987). Somatosensory detection of microgeometry, macrogeometry and kinesthesia after localized lesions of the cerebral hemispheres in man. Brain Research Reviews, 12, 43-94.

Roland, P. E., \& MorTENSEN, E. (1987). Somatosensory detection of microgeometry, macrogeometry and kinesthesia in man. Brain Research Reviews, 12, 1-42.

Sadato, N., Pascual-Leone, A., Grafman, J., Deiber, M.-P., IbaNEZ, V., \& HALLETT, M. (1998). Neural networks for Braille reading by the blind. Brain, 121, 1213-1229.

Sadato, N., Pascual-Leone, A., Grafman, J., Ibanez, V., Deiber, M.-P., Dold, G., \& Hallett, M. (1996). Activation of the primary visual cortex by Braille reading in blind subjects. Nature, 380, 526-528.

SaGr, D., \& TANNE, D. (1994). Perceptual learning: Learning to see. Current Opinion in Neurobiology, 4, 195-199.

SaTHIAN, K. (1989). Tactile sensing of surface features. Trends in Neurosciences, 12, 513-519.

Sathian, K., Goodwin, A. W., John, K. T., \& Darian-Smith, I. (1989). Perceived roughness of a grating: Correlation with responses of mechanoreceptive afferents innervating the monkey's fingerpad. Journal of Neuroscience, 9, 1273-1279.

Sathian, K., \& Zangaladze, A. (1996). Tactile spatial acuity at the human fingertip and lip: Bilateral symmetry and inter-digit variability. Neurology, 46, 1464-1466.

Sathian, K., \& Zangaladze, A. (1997). Tactile learning is taskspecific but transfers between fingers. Perception \& Psychophysics, 59, 119-128.

Sathian, K., \& Zangaladze, A. (1998). Perceptual learning in tactile hyperacuity: Complete intermanual transfer but limited retention. Experimental Brain Research, 118, 131-134.

Sathian, K., Zangaladze, A., Hoffman, J. M., \& Grafton, S. T. (1997). Feeling with the mind's eye. NeuroReport, 8, 3877-3881.

Sergent, J., Ohta, S., \& MacDonald, B. (1992). Functional neuroanatomy of face and object processing: A positron emission tomography study. Brain, 115, 15-36.

Shimizu, Y., Saida, S., \& Shimura, H. (1993). Tactile pattern recognition by graphic display: Importance of 3-D information for haptic perception of familiar objects. Perception \& Psychophysics, 53, 43-48.

Summers, D. C., \& Lederman, S. J. (1990). Perceptual asymmetries in the somatosensory system: A dichhaptic experiment and critical review of the literature from 1929 to 1986. Cortex, 26, 201-226.

van Boven, R. W., \& Johnson, K. O. (1994a). The limit of tactile spatial resolution in humans: Grating orientation discrimination at the lip, tongue and finger. Neurology, 44, 2361-2366. 
VAN BOVEN, R. W., \& JohnSON, K. O. (1994b). A psychophysical study of the mechanisms of sensory recovery following nerve injury in humans. Brain, 117, 149-167.

Westheimer, G. (1977). Spatial frequency and light-spread descriptions of visual acuity and hyperacuity. Journal of the Optical Society of America, 67, 207-212.

Wheat, H. E., Goodwin, A. W., \& Browning, A. S. (1995). Tactile resolution: Peripheral neural mechanisms underlying the human capacity to determine positions of objects contacting the fingerpad. Journal of Neuroscience, 15, 5582-5595.

Zangaladze A., Epstein, C. M., Grafton, S. T., \& Sathian, K. (1999). Involvement of visual cortex in tactile discrimination or orientation. Nature, 401, 587-590.

\section{NOTES}

1. Causes of blindness in the early-blind group were retinopathy of prematurity (6), optic nerve hypoplasia (2), congenital cataracts (4), trauma (1), and congenital blindness of unknown etiology (2). In the late-blind group, blindness was due to congenital cataracts associated with glaucoma (5), retinal detachment (1), trauma (1), and unknown causes (2).

2. Two sighted subjects were excluded because of a history of carpal tunnel syndrome. One blind subject was excluded because of a prior stroke, and another was excluded due to concurrent deafness and cognitive impairment.

3. Paired $t$ tests comparing performance between Sessions 3 and 4 of Experiment 1 yielded the following results: sighted subjects, dominant hand, $p=.92$; blind subjects, dominant hand, $p=.58$; blind subjects, nondominant hand, $p=.9$.

4. A few subjects could not complete four sessions, and an initial DL could not be obtained due to incomplete data in 1 normal subject (S33, Table 2).

5. Values of $p$ on paired $t$ tests for these comparisons between Sessions 3 and 4 of Experiment 2 were as follows: sighted subjects, dominant hand, $p=.51$; blind subjects, dominant hand, $p=.38$; blind subjects, nondominant hand, $p=.29$.

6 . Values of $p$ on paired $t$ tests for these comparisons between Sessions 1 and 2 of Experiment 3 were as follows: sighted subjects, dominant hand, $p=.32$; sighted subjects, nondominant hand, $p=.76$; blind subjects, dominant hand, $p=.14$; blind subjects, nondominant hand, $p=.76$.

(Manuscript received April 7, 1998; revision accepted for publication January 6, 1999.) 